\title{
Optimizing Fuel Cell Materials Through Electron Microscopy and Microanalysis
}

\author{
D.A. Cullen, H.M. Meyer, K.S. Reeves, D.W. Coffey, and K.L. More
}

Materials Science \& Technology Division, Oak Ridge National Laboratory, Oak Ridge, TN 37831

Polymer electrolyte membrane fuel cells (PEMFCs) are under intense research and development for transportation applications. Vehicles powered by hydrogen fuel cells provide a driving experience comparable to today's gas powered vehicles, while producing only water and heat as byproducts.[1] However, key cost and performance targets must be met before this technology can advance into the pre-commercial phase.[2] Electron microscopy continues to play an important role in the development of fuel cell materials and processes aimed towards meeting these critical targets. In the present work, we highlight recent collaborative efforts with industrial and government partners aimed at driving PEMFCs towards real world application.

The wide range in materials used in the fuel cell stack, from the pivotal nanoparticle Pt group metal (PGM) catalysts to the proton exchange membrane, requires an equally wide range of microscopy preparation and analysis techniques. The microscopy methods employed include aberrationcorrected imaging, energy dispersive X-ray spectroscopy, X-ray photoelectron spectroscopy (XPS), and low voltage, cryogenic microscopy.

Fig. 1 shows a few of the many microscopy sample preparation methods, which have been used to prepare a variety of fuel cell components for analysis, including conventional carbon-supported PGM catalysts, membrane electrode assemblies (MEAs), gas diffusion layers, and nanostructured thin films, for analysis by electron microscopy and complementary techniques (Fig. 2). In some cases, additional methods such as drop casting, diamond knife ultramicrotomy, and focused ion beam (FIB) milling, were modified for specific material constituents. Two such examples were ultra-low angle microtomy for conducting cross-sectional XPS measurements of MEAs, and unique FIB lift-outs for $360^{\circ}$ tomography.

Quantitative microscopy and spectroscopy methods were employed at various length scales to not only provide microstructural and compositional insight into as-grown/assembled materials, but to also examine the impact of real-life fuel cell vehicle operations, such as potential cycling and transient events, on the fuel cell components. A few such examples are presented in Fig. 3. EDS methods were used to visualize multiple catalyst layers on nanostructured supports, to observe coreshell nanoparticle structures, and to quantify Pt dissolution within the MEAs. TEM imaging was used to explore the impact of graphitization on carbon corrosion. Finally, low voltage, cryo-electron microscopy was used to image and analyze thin, proton-conductive ionomer layers in electrodes.[3]

\section{References}

[1] F.T. Wagner. B. Lakshmanan, M.F. Mathias, J. Phys. Chem. Lett. 1 (2010) 2204.

[2] M.K. Debe, Nature 486 (2012) 43.

[3] Research supported by (1) the Fuel Cell Technologies Program, Office of Energy Efficiency and Renewable Energy, U.S. Department of Energy under Award Number DE-EE0000456 and (2) ORNL's ShaRE User Facility, Office of Basic Energy Sciences, U.S. Department of Energy. 

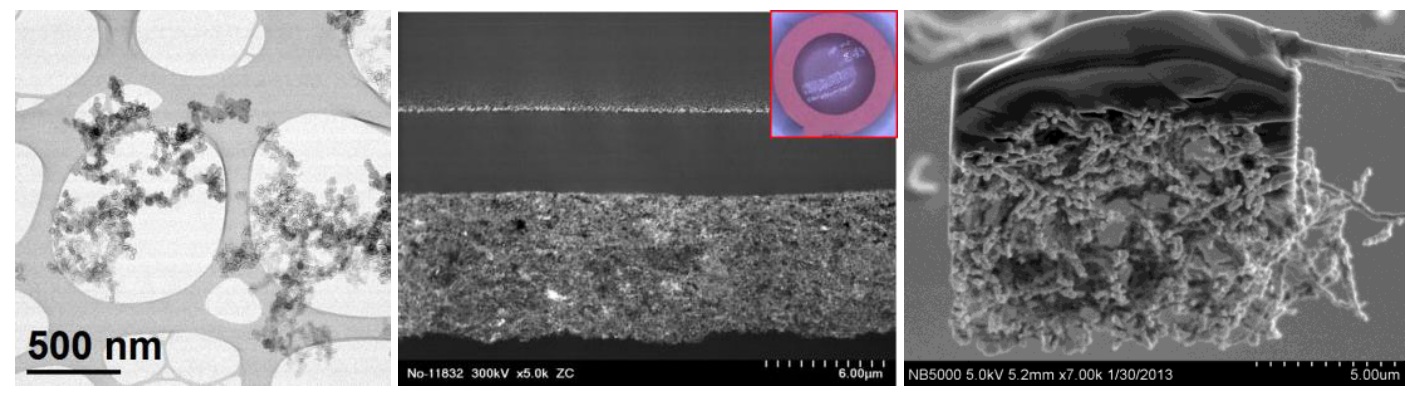

FIG. 1. Images of different sample preparation methods employed to study fuel cell material components by electron microscopy. From left to right: drop casting on a lacey carbon-coated $\mathrm{Cu}$ grid, MEA ultramicrotomy, and FIB lift-out.
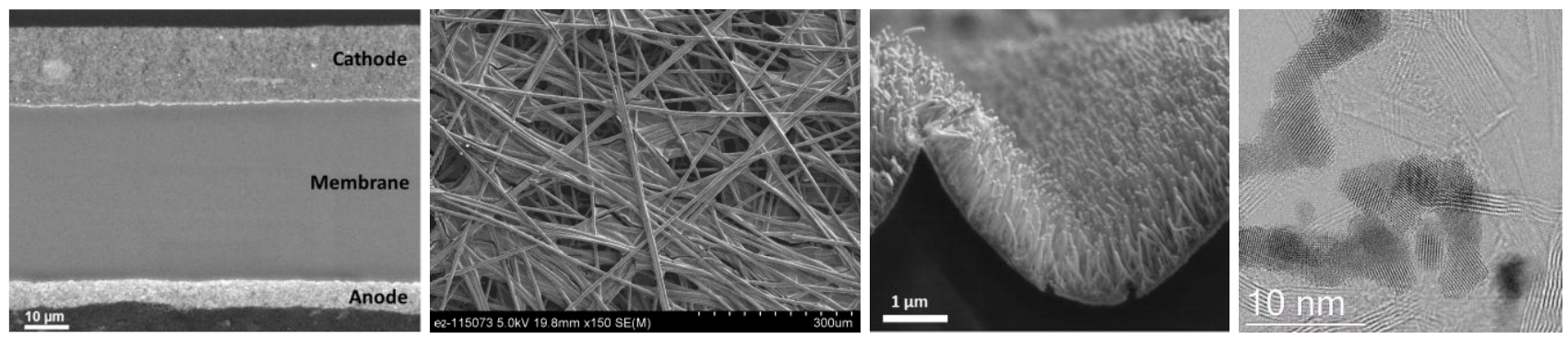

FIG. 2. SEM images of a MEA cross-section, gas diffusion layer, Pt nanostructured thin film, and BF-STEM image of Pt nanoparticle catalysts on low surface area carbon.
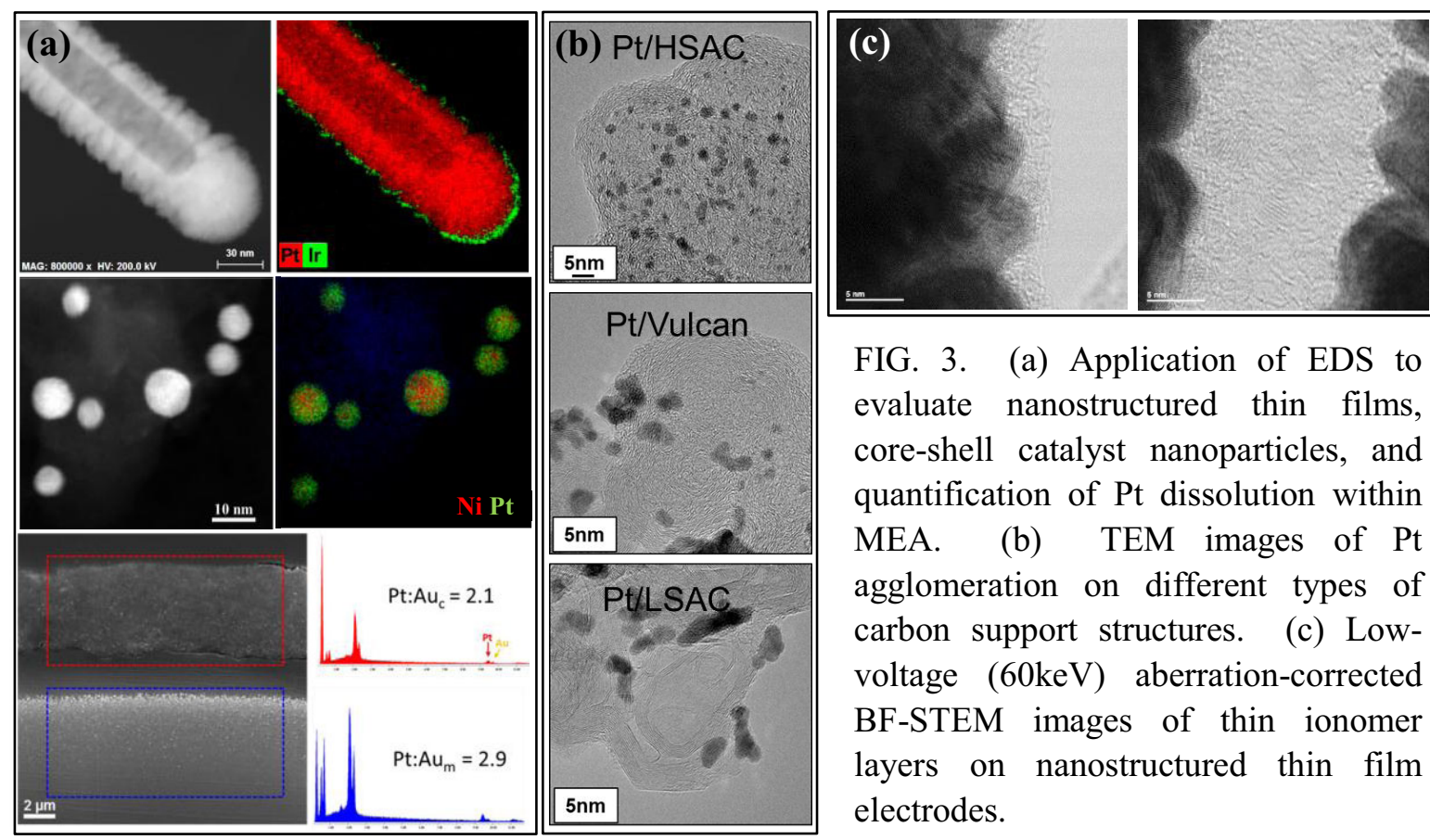

FIG. 3. (a) Application of EDS to evaluate nanostructured thin films, core-shell catalyst nanoparticles, and quantification of $\mathrm{Pt}$ dissolution within MEA. (b) TEM images of $\mathrm{Pt}$ agglomeration on different types of carbon support structures. (c) Lowvoltage $(60 \mathrm{keV})$ aberration-corrected BF-STEM images of thin ionomer layers on nanostructured thin film electrodes. 